\title{
Zur deutschen Übersetzung des ,Circa instans' im ,Medizinischen Kompendium des Juden von Salms'
}

\section{German Translation of ,Circa instans' in the ,Medical Compendium by the Jew of Salms'}

\author{
Lenka Vaňková - Václav Bok
}

\begin{abstract}
The medical compendium by the Jew of Salms, dating from the first half of the 15th century, includes various types of medical texts; one of them is a German translation of one of the bestknown medieval herbaria, known under the title 'Circa instans'. A complete translation of this text only exists in one of the three surviving manuscripts (manuscript C4a at Zurich University Library). The article discusses the specific features of the translation method used by the Jew of Salms and demonstrates the unique nature of this translation in comparison with a different translation (part of manuscript 1224 at Leipzig University Library).
\end{abstract}

\section{Keywords}

Circa instans; medieval herbaria; medieval medical texts; the Jew of Salms; translations; German 


\section{Einleitung: das ,Circa instans'}

Das ,Circa instans` wird - nicht ganz zu Unrecht - neben dem ,Macer floridus` für das wichtigste Kräuterbuch des Mittelalters gehalten (vgl. Schnell 2003: 21) ${ }^{1}$ und zu denjenigen Werken gerechnet, die die „Verselbständigung des abendländischen Apothekerstandes“ (Keil 1978: 1282) beeinflusst haben. ${ }^{2}$ Diese Sammlung von Beschreibungen einfacher Drogen, die nach ihrem Anfangsbuchstaben in die einzelnen nach dem Alphabet geordneten Kapitel eingeteilt sind, wurde jahrhundertelang als das grundlegende praktische Handbuch von Ärzten und Apothekern genutzt.

Das ,Circa instans“ ist zwischen den Jahren 1150 und 1170 in Salerno entstanden. ${ }^{3}$ Salerno war im 12. Jh. ein wichtiges Zentrum der Medizin, in dem sich die antike Tradition und das arabische Kulturgut getroffen haben (vgl. Damm 1939: 4). Das spiegelt sich im ,Circa instans، wider. ${ }^{4}$ Man findet hier zahlreiche Drogen fremder Provenienz, sodass man sagen kann, dass das ,Circa instans“ das Eindringen arabischer bzw. indischer Drogen und ihre Anwendung bei den Heilverfahren im Abendland unterstützt hat.

Von seiner weiten Verbreitung zeugt die Tatsache, dass die lateinische Fassung heute in etwa 240 Handschriften $^{5}$ zur Verfügung steht. Diese weisen eine hohe Variabilität auf, wobei in der bisherigen Forschung meist zwischen der „Kurz- und Langfassung“ unterschieden wurde. Zu den ältesten Überlieferungsträgern des ,Circa instans“ wird der Text in der Handschrift Nr. 674 aus Erlangen gerechnet, der im 13. Jh. entstanden ist. Dieser Text repräsentiert die Kurzfassung. Er steht uns in zwei Editionen zur Verfügung: Einer älteren von Wölfel aus dem Jahr 1939 und einer neueren, die 2015 Goehl zusammen mit der Übersetzung des Textes in die deutsche Gegenwartssprache publiziert hat. Dieser Text, der wahrscheinlich der Urfassung sehr nahe steht, enthält 252 Drogenmonographien. Goehl hat in seine Edition noch 6 weitere Drogen einbezogen, deren Beschreibungen sich in der New Yorker Handschrift Ms. 0011 befinden und die wahrscheinlich zum ursprünglichen Text gehörten. Was die sog. Langfassung betrifft, war einer der ältesten Textzeugen im Breslauer, Codex Salernitanus', einer medizinischen Sammelhandschrift des ausgehenden 12. Jh., enthalten. Die Handschrift ist seit dem Ende des 2. Weltkriegs verschollen. Nach Holler (1940), der in seinem Buch einzelne ausgewählte Monographien abgedruckt und bei allen übrigen lediglich Hinweise auf ihre Provenienz

1 Zum Unterschied vom ,Macer“ enthält das ,Circa instans‘ jedoch nicht nur Informationen über Heilpflanzen, sondern auch über Drogen aus dem Tier- und Mineralbereich.

2 Nach Keil (1978: 1282) haben neben ,Circa instans“ noch das „Antidotarium Nicolai“ und Platearius“ ,Liber iste“„durch Auswahl und Anlage wesentlich zur Standardisierung sowie Lagerfähigkeit der Arzneimittel“ beigetragen. Alle diese Werke sind um Mitte des 12. Jh. entstanden.

3 Die Autorschaft wird manchmal einem Angehörigen der bedeutenden Familie Platearii, Matthaeus Platearius, zugeschrieben (vgl. z. B. Mayer 2014: 135, Goehl 2015: 7).

4 Nach I. Ventura (2016: 398) „the CI (,Circa instans`) is not an easy melting pot, but the mirror of a complicated clash of traditions“. Sie weist darauf hin, dass der ,Liber de gradibus" des Konstantin von Afrika beim Verfassen des ,Circa instans' eine besondere Rolle spielte, weil danach „the general structure of entries, the selection of the natural substances, the technical vocabulary“ (ebd.) übernommen wurden.

5 Davon ist in 138 der mehr oder weniger komplette Text überliefert (vgl. Ventura 2016: 339). 
angeführt hat, gab es in der Handschrift über 450 Monographien. ${ }^{6}$ Zu demselben Überlieferungsstrang gehört u. a. auch ,Tractatus de herbis‘ aus der Londoner British Library (Egerton 747), vgl. Ventura (2009).

Wie einflussreich das ,Circa instans“ war, bezeugen auch zahlreiche Verweise auf das Buch oder Übernahmen von Teilen des Buches in andere Kräuterbücher bzw. Drogenkompendien sowie Übersetzungen in mehrere Landessprachen: ins Englische, Französische, Italienische, Niederländische, Dänische, Hebräische, Serbische, Katalanische (vgl. Ventura 2016: 339). Das ,Circa instans“ wurde auch ins Deutsche übersetzt. Neben Übersetzungen aus dem niederdeutschen (niederfränkischen) Sprachraum (vgl. Keil 1978: 1283) sind auch hochdeutsche Bearbeitungen belegt.

\section{Zu den bisher bekannten hochdeutschen Übersetzungen des "Circa instans'}

Eine ostmitteldeutsche Übersetzung des ,Circa instans' steht in der Leipziger Handschrift Cod. 1224 (vgl. Keil 1978: 1284), die als ,Leipziger Drogenkompendium‘ bekannt ist, ${ }^{7}$ zur Verfügung. Die Edition des ,Leipziger Drogenkompendiums' liegt noch nicht vor. Im Jahre 1939 hat Walter Damm die Arbeit mit dem langen Titel ,Die einzige bisher bekannte deutsche Fassung des Buches Circa instans (de simplicibus) nach einer Handschrift des 15. Jahrhunderts (Leipzig, Universitätsbibliothek Nr. 1224)‘ vorgelegt. In dieser Arbeit finden sich jedoch leider nur Verzeichnisse der im Text vorkommenden Drogen, die am Anfang jedes dem betreffenden Buchstaben gewidmeten Kapitels stehen, und im vollen Wortlaut nur diejenigen Monographien, die in der Erlanger lateinischen Fassung im Kodex 674 nicht enthalten sind.

Insgesamt kommen im ,Leipziger Drogenkompendium“ 328 Drogenmonographien vor (vgl. Mayer 2000: 208), ${ }^{8}$ wobei jedoch einige nicht aus dem ,Circa instans` stammen, sondern aus anderen Quellen. Nach Mayer (2000) hat der Autor/Übersetzer auch aus dem „Macer', dem ,Liber de gradibus“ des Konstantin von Afrika sowie aus dem ,Aggregator ${ }^{‘ 9}$ geschöpft, wobei er oft in einer Drogenmonographie mehrere Quellentexte kombiniert hat.

Eine bairisch-österreichische Übersetzung des ,Circa instans' haben Palmer und Speckenbach in der sog. ,Petroneller Handschrift ${ }^{60}$ entdeckt. Diese Handschrift, die nach

6 Die verschiedenen Versionen der „Langfassung“ bestehen nach Ventura (2016: 340) aus 500-900 Drogenmonographien.

7 Vgl. das gleichnamige Stichwort im Verfasserlexikon von Mayer (2004: 912-915).

8 Die Angaben über die Anzahl der Drogenmonographien gehen bei Mayer auseinander. Im ,Verfasserlexikon“ gibt er 337 Drogenmonographien an und macht darauf aufmerksam, dass „die bisherige Literatur andere Zahlen" nennt (Mayer 2004: 912). Das Problem besteht darin, dass in Drogenverzeichnissen manchmal solche angeführt sind, die im Text nicht behandelt werden und umgekehrt, sodass nur eine genaue Sichtung der Handschrift zuverlässige Angaben bieten kann.

9 Als ,Aggregator` wird eine Arzneimittellehre bezeichnet, die „fälschlicherweise dem syrischen Arzt Johannes Serapion zugeschrieben wurde“ und die teilweise „aus der arabischen Medizin“ kommt. Sie ordnet die Drogen nicht nach den Namen, sondern nach den Intensitätsgraden (vgl. Mayer 2014: 135-136).

10 Diese Handschrift wurde im Auftrag von Lamprecht Kripp, einem Angehörigen einer reichen Tiroler 
dem Schloss Petronell bei Wien benannt wurde, enthält u. a. auch eine Langfassung des ,Circa instans“, die um Mitte des 15. Jh. geschrieben wurde und der später (wahrscheinlich um 1482) auf eingelegten Blättern synoptisch eine deutsche Übersetzung beigefügt wurde.

Es war bekannt, dass eine deutsche Version des ,Circa instans' auch im Kompendium des Juden von Salms vorliegt (vgl. Keil 1961: 136, 140f.). Man hat jedoch angenommen, dass sich im Kompendium nur eine Teilübertragung des ,Circa instans' befindet (vgl. Keil 1978: 1284). Unsere Untersuchung des Medizinischen Kompendiums des Juden von Salms ${ }^{11}$ die im Rahmen des Projekts der Forschungsagentur der Tschechischen Republik durchgeführt wird, hat gezeigt, dass in einer der drei verfügbaren Überlieferungen des Kompendiums eine komplette Übersetzung des ,Circa instans` belegt ist.

Im Folgenden werden diese Übersetzung, ihre Anlage und ihr Inhalt, vorgestellt und die Strategie des Juden von Salms bei der Übersetzung bzw. Übertragung des ,Circa instans'-Textes ins Deutsche charakterisiert. Zuerst aber gehen wir auf die Frage ein: Wer war der Jude von Salms?

\section{Der Jude von Salms und sein ,Medizinisches Kompendium'}

Vom Juden von Salms ist wenig bekannt. Einige Informationen über sein Leben bieten eigene Bemerkungen in seinem umfangreichen Kompendium. So schreibt er z. B. auf Bl. 270ra:

Ich Hesse der Jüdde von Sallniß, eyn geselle in medicina vnd cyrologia [...] gepfleget erczenie myt eren woil sesz vnd sechczyg jare [...], gedyenet lantherren, konigen, herczogen, graffen, ryttern, knechten vnd gemeynem volcke.

An mehreren Stellen seines Kompendiums nennt sich der Autor Hesse (z. B. K 153rb, K 167va, K 168va), manchmal auch Hesse der Iode (K 1ra bzw. Hesse der Jüdde Z 1ra, Hesse der Jüde K 147va) und Hesse von Salniß (K 42ra bzw. Hesse von Salynß Z 38ra) sowie Heße der Judde von Salm $\beta$ (E 271rb), vgl. Bok (2014: 66). ${ }^{12}$

In seinem Kompendium präsentiert er sich als erfahrener, selbstbewusster Arzt, der gut ausgebildet und belesen ist. Davon zeugen zahlreiche Verweise auf anerkannte medizinische Autoritäten wie Hippokrates, Galen, Avicenna, Albertus Magnus oder Rhasis sowie Vertreter der jüdischen Medizin wie Moses Maimonides und Isaak Israeli. Er zitiert auch seine Zeitgenossen, was beweist, dass er in den medizinischen Kreisen seiner

\footnotetext{
Bürgerfamilie, im 15. Jh. geschrieben. Sie besteht aus lateinischen, deutschen und lateinisch-deutschen Texten, die in zwei Phasen - um 1450 und um 1482 entstanden sind (vgl. Palmer - Speckenbach 1990: 4).

11 Es handelt sich um das Projekt GA18-17754S Lékařské kompendium Žida ze Salmsu - historický kontext jazykový rozbor - edice [Das Medizinische Kompendium des Juden von Salms - historischer Kontext - sprachliche Analyse - Edition]. Im Rahmen dieses Projekts ist auch der vorliegende Beitrag entstanden.

12 K steht für Krumau, E für Erlangen, Z für Zürich.
} 
Zeit über zahlreiche Kontakte verfügte. Er scheut sich auch nicht, auf seine eigenen therapeutischen Erfolge aufmerksam zu machen (vgl. Vaňková 2017: 48-49).

Der Jude von Salms verrät auch, dass ihn Graf Johann V. von Sponheim (um 13591437) zum Verfassen seines Sammelwerkes anregte, bei dem er die letzten Jahre seines Lebens verbrachte. Der Anstoß kam wahrscheinlich bei einem Treffen auf der Burg Grevenburg im Jahre 1427. Vor dem letzten Teil des Buches ist das Datum 20. Februar 1430 vermerkt, sodass man voraussetzen kann, dass kurz danach das Werk abgeschlossen war, wobei der Jude von Salms nach Angaben in seinem Buch zu jener Zeit schon über 80 Jahre alt gewesen sein dürfte (vgl. Bok 2014: 68).

Das medizinische Kompendium des Juden von Salms liegt zurzeit in drei Abschriften vor. Die älteste davon ist die Handschrift Nr. 448 aus Český Krumlov/Krumau, die nach der Analyse der vorhandenen Filigrane - spätestens Ende der 40er Jahre des 15. Jh. geschrieben wurde, also kurz nach der Niederschrift des Originaltextes. Für den zweitältesten Textzeugen wird die Züricher Handschrift C 4a gehalten, die nach der Angabe auf dem letzten Blatt wahrscheinlich um 1462 entstanden ist. Die dritte Abschrift (Hs. B 34), die sich in der Universitätsbibliothek in Erlangen befindet, ist wahrscheinlich Ende des 15. Jh. geschrieben worden (vgl. Bok 2014: 71-72).

Alle Textzeugen haben einen sehr ähnlichen Umfang - die Züricher Handschrift enthält 282 Folios, die Erlanger 274, die Handschrift von Krumau, deren Ende fehlt, 255 Folios.

Was den Inhalt betrifft, besteht das Kompendium aus mehreren Teilen, wobei der komplette Text nur in der Züricher Handschrift vorhanden ist. Im ersten Teil findet man eine Übersetzung aus den Schriften von Avicenna, auf die im zweiten Teil eine Übersetzung des Arzneibuches des angesehenen französischen Arztes Johannes Jacobi von Montpellier $(\uparrow 1384)$ folgt, die durch zusätzliche Passagen ergänzt ist. Das dritte Buch ist eine Chirurgie, die eine Kompilation mehrerer Quellen darstellt. Im vierten Teil ist ein Gesundheitsregimen zu finden. Der folgende Teil enthält die Übersetzung des lateinischen Arzneidrogenbuches ,Circa instans'. In der Krumauer Handschrift ist der Text mitten in ,Circa instans‘ abgebrochen, in dem Textzeugen aus Erlangen ist das ,Circa instans‘ gekürzt. Die Handschriften aus Erlangen und Zürich bieten nach dem Text des ,Circa instans` eine weitere Ergänzung zum Gesundheitsregimen, in der unter dem Aspekt der Humoralpathologie verschiedene für das Leben des Menschen wichtige Materien und Nahrungsmittel charakterisiert werden. Während in der Handschrift aus Erlangen nach der Behandlung der Öle der Text beendet wird, werden in der Züricher Handschrift noch verschiedene Getreidesorten, Fleischsorten, Pelzsorten erörtert und abschließend noch einige Rezepte angeführt (vgl. Bok 2014: 69-70).

Da die früheren Beschreibungen des Kompendiums des Juden von Salms von dem Textzeugen aus Erlangen (vgl. Keil 1978: 1284) bzw. Krumau (Bok 2014: 68-70) ausgegangen sind, in denen mehrere Kapitel des ,Circa instans' fehlen, hat man angenommen, dass sich im Kompendium vielleicht nur eine Teilübertragung des ,Circa instans befindet. Der Textzeuge aus Erlangen enthält insgesamt 183 Drogenmonographien. Inmitten des Textes wurden die Kapitel zu den Buchstaben M bis R ausgelassen und die Kapitel zu L und S sind unvollständig. Der Krumauer Text beinhaltet lediglich 99 Drogenmonographien, weil dort nur die Kapitel zu den Buchstaben A bis D vorkommen. 
Das ,Circa instans‘ in der Züricher Handschrift enthält 293 Drogenmonographien, hier sind alle Buchstaben vertreten, es handelt sich also um eine vollständige Übersetzung bzw. Überarbeitung der lateinischen Urvorlage. Um welche Vorlage es sich handelt, lässt sich jedoch im jetzigen Stand der Forschung und auch angesichts der Menge der überlieferten Varianten des ,Circa instans'-Textes nicht genau bestimmen.

\section{Zu Anlage und Inhalt des ,Circa instans' im Kompendium des Juden von Salms}

Aus dem Vergleich der Anzahl der Drogenmonographien im Kompendium des Juden von Salms mit der in der lateinischen Fassung in der Handschrift Nr. 674 aus Erlangen (also der autornahen Kurzfassung des ,Circa instans') ergibt sich, dass beim Juden von Salms etwa 40 Drogenmonographien mehr vorliegen. Etwa die Hälfte der zusätzlichen Kapitel ist auch im ,Leipziger Drogenkompendium“ enthalten, sodass man fragen kann, ob sie auf dieselbe Quelle zurückgreifen. Zum Beispiel wurde die Monographie Arnoglossa in dem Leipziger Drogenkompendium aus ,Liber de gradibus‘ des Konstantin von Afrika übersetzt, wie Johannes Mayer nachgewiesen hat (2000: 257-258). Dieses Kapitel, das in der lateinischen Fassung aus Erlangen nicht vorhanden ist, findet man auch im ,Circa instans'-Text des Juden von Salms. Beide Arnoglossa-Texte weisen einen hohen Ähnlichkeitsgrad auf, auch wenn man geringe Unterschiede sowohl im Inhalt (vgl. die Angabe der Primärqualitäten, kleinere Abweichungen im Bereich der Indikationen) als auch in der Wortwahl feststellen kann (vgl. z. B. das lateinische sive im LDk - im Züricher Text das ist, trughe (trocken) - dürr, wunden - schaden, czene vleisch - biler, die Krankheit Ergotismus wird im LDk als heiligher vure bezeichnet, im Züricher Text als sant Antonius feuer).

Arnoglossa, wegebreite sive plantago major, ist kalt vnd trughe in dem ij grate, vnde heizet ouch plantago vnd quinqueneruia. Vnd ist nutze dy wunden zu trughene und reinighende, vnd vorzeret yre vnreinikeit, vnd sterket vnde wederstet dem heilighen vure, das is nicht vmme wandert alle den lip. Vnd ist gut weder dy emorroydas vnde weder irkeldet alle hiczcze von der zcu burnunghe. Vnd ist nutcze emoptoycis vnd dissenterijs vnde dy den vluz der menstrua haben. Vnd heilet dy wunden der lunghen. Vnde syn worczele in wasser gecochet semftighet dy wetaghe der czene ist dazs man den munt mit dem wasser geweschet. Galienus spricht, das saff quinqueneruie ist gut weder dy bestopphunge der nyren. Dyacsorides spricht, das is varwet dy swarczen vnd truckele vlecken vnd allermeist syn same zczu
Arnoglossa, daz ist wegebreyt, vnd ist kalt vnd dürre inne dem drytten grade. Vnd ist czweyerley, daz eyn ist genant quinque nerne, daz ander ist genant plantago mynoris. Vnd helffent zü mal sere vnd drückent alle schaden vnd reynigent vnd dünt abe vbeln geschmacke vnd sterckent daz mylcze vnd hylffe[n]t zü male sere. Zü sant Anthonius füre vnd sint aüch gut zü amorides vnd zü vberger hytze dye würczel gesotten yn waszer vnd do myt den mont gewassen, ist güt zü allem wee der czene. Vnd sprychet Galienus, waszer gebrant von quinque nerne vnd ist güt zu swylst der nyeren vnd zu swarczen flecken, als reflecken reyniget vnd lütert ez. Vnd der same ist noch beszer. Der bresten, der yn den bylren ist, nymmet yz auch abe. 
reben weder heldet das blut des czene vleisches. Aüch wysze seüffe geküwet ist güt zu dem Vnd ro zcu kuwet benimmet dy wethage der cze- selben... ne der czene...

(Leipzig, Hs. 1224, 31rb-31va, nach Mayer 2000: (Zürich, Hs. C 4a: 213vb, Vaňková - Bok in Vorbereitung)

257-258)

\section{Arnoglossa-Monographie - Proben von äquivalenten Textstellen}

Bei der Identifizierung von Drogen in dem ,Circa instans' begegnet man manchmal Problemen, die verschiedene Ursachen haben können.

Zahlreiche Drogennamen (sowie manche anderen Fachausdrücke) sind fast zur Unkenntlichkeit verballhornt. Diese Schwierigkeiten hängen zum großen Teil mit der geringen Kompetenz des Schreibers zusammen, der sich in der Problematik wenig auskannte und die Vorlage nicht gut verstanden hat. Zum Teil wirkten sich aber auch sprachliche Gewohnheiten des Autors, also des Juden von Salms aus, der wahrscheinlich französischer Abstammung war oder zumindest aus einem französisch-deutschen gemischten Sprachgebiet stammte. ${ }^{13}$ Als Gallizismus lässt sich zum Beispiel die Anführung von cpica im Verzeichnis zu dem Buchstaben C gewidmetem Kapitel deuten, wobei es sich um Spica nardi, d. h. Indische Narde/Nardenähre handelt.

Auch die genaue Anzahl der behandelten Drogen musste nach dem Vergleich der einzelnen Texte revidiert werden, denn es passiert mehrmals, dass dieselbe Droge wiederholt an unterschiedlichen Stellen beschrieben wird.

Unter dem Buchstaben C wird zweimal - auf Bl. 218va-218vb (a) und auf Bl. 227vb228ra (b) - dieselbe Pflanze, Chelidonium majus/Schöllkraut (im Text Celydonia), eingeordnet. Die beiden Celydonia-Monographien weichen teilweise voneinander ab. Im Text (a) fehlt die Einleitung, die im lateinischen Text vorhanden ist (vgl. Goehl 2015: 75), mit dem Hinweis auf den Unterschied zwischen der großen und der kleinen Sorte, der schon von Konstantin hervorgehoben wurde (Vnd ist czweyerley, grosz und cleyne. Aber die groiß hait mer dogent wan dye kleyn. Vnd vorware sprychet Constantinus, keyn ist als güt als dye grosz), weiter die Angabe über die Lagerungszeit (vnd weret dry jare) und die Anmerkung, dass als Heilmittel die Wurzel von Schöllkraut verwendet wird (Vnd wan man heyschet yn arczenie celydonia, so prüffet, daz man dye würczel meynet). Im Indikationsbereich stimmen zwar die Texte (a) und (b) überein, die Formulierungen sind jedoch nicht exakt identisch, z.B.:

Zü colica passio sudt celidonia myt gudem win. Vnd nym eynen badem [!] swamp vnd düncke den in dye decoccio vnd lege off den nabel oder den büche, daz ist zü male güt (a)

$\mathrm{Zu}$ colica passio nym celidonia crut vnd syede dye myt wyn vnd düncke eynen bade swamp in dye decoctio vnd lege yn off den wethüm als warm (b).

13 Der Jude von Salms entschuldigt sich an mehreren Stellen seines Kompendiums für seine mangelhaften Deutschkenntnisse. So schreibt er z. B. auf dem Bl. 42vb: „wie wol daz mir doch dasselbe etwe witder vnd swere was, deshalben, daz ich die dutsche sprache nit wöl vnd nach myme willen kann." 
In der Celydonia-Monographie auf Bl. 218vb erscheint noch eine zusätzliche Indikation, die weder im (b)-Text, noch in der lateinischen Version des ,Circa instans“ (vgl. Goehl 2015: 75) zu finden ist: Zü fynsternisse vnd nebel der auwen ond stare celydonien waßer myt anderley crüt waszer, daz ist dar zü güt. Die Tatsache, dass in den beiden anderen Abschriften des Kompendiums des Juden von Salms die Monographie über Schöllkraut zweimal - genau wie im Züricher Text - vorkommt und gleichzeitig im Verzeichnis der mit C beginnenden Drogen Chelidonium nur einmal angeführt wird, zeugt davon, dass diese Lage dem Originaltext entspricht. Dies lässt sich vielleicht auf das hohe Alter des Juden von Salms und die damit verbundene Unaufmerksamkeit zurückführen, die dazu führte, dass er ein- und dieselbe Droge zweimal bearbeitet hat.

Ebenso erscheint die Monographie zu Cassia lignea, d. h. zu der Zimtrinde (im Text cassia lygna/cassia ligna) zweimal an unterschiedlichen Stellen des dem Buchstaben C gewidmeten Kapitels, wobei diese Pflanze auch im Verzeichnis zweimal angeführt ist. Auch in den zwei anderen Abschriften des Circa instans findet man diese Wiederholung. Die cassia lignea-Monographien weichen jedoch in hohem Maße voneinander ab. Der Text auf Bl. 219rb beinhaltet lediglich die Information von den Eigenschaften (ist hytzig vnd dürre in dem dritten grade) und eine Übersicht der Indiktionen, bei denen sie verwendet werden kann. Dagegen weist die andere Monographie (Bl. 222vb-223ra), besonders am Anfang des Textes, eine starke Ähnlichkeit mit der lateinischen Vorlage auf. Die ersten Sätze stellen eine getreue Übersetzung der lateinischen Einleitung dar. Im Indikationsbereich fasst sich jedoch der Jude von Salms viel kürzer, sodass es sich eher um eine Zusammenfassung der Indikationen als um eine Übersetzung handelt.

Sogar an drei unterschiedlichen Stellen ist eine Spica-Monographie anzutreffen. Den ersten Text über Spica nardi, d. h. Indische Narde/Nardenähre, hat der Jude von Salms am Anfang des C-Kapitels untergebracht. Im Verzeichnis beginnt diese Bezeichnung mit c (also cpica), im darauffolgenden Text wird vor spica noch eine A-Initiale (Aspicanardi) vorangestellt. Der Text im C-Kapitel (Bl. 218rb-218va) ist inhaltlich identisch mit der Abhandlung über die indische Narde im S-Kapitel auf Bl. 260vb-261ra. Im Wortlaut beider Texte kann man aber zahlreiche Abweichungen finden, die beweisen, dass der Jude von Salms nicht bloß den Text noch einmal abgeschrieben hat, sondern es wird geradezu der Eindruck erweckt, dass er denselben Text zweimal übersetzt/bearbeitet hat, vgl.:

Vnd eyner fraüwen, der yr czyt flüszet an vnderlaisze, neme spicanardi vnd syeden den in waszer vnd syecze dar yn myt an den nabel. Aüch hytczige swylst oder postemen in dem magen vnd in dem matricis heylet er. Den dampf von spycanardi inphangen inne dye matrix offent dye nature von beshlossünge matricis vnd erwecket fraüwen sychtage. (218rb-218va)
Vnd eyn fraüwen, dye yr czyt vberich hayt, spicanardi gesotten in waszer vnd sie dar yn geseszen, ist sere güt. Aüch hytzige geswilst vnd postemen in dem magen vnd matrix nymmet ez abe. Sin raüche offent virstoppenisse matricis vnd erwecket fraüwen sychtage. (260vb-261ra)

\section{Spica-Monographie - Proben von äquivalenten Textstellen}


Die dritte Monographie über spica (Bl. 261rb-261) unterscheidet sich von den vorangehenden. Ihr Anfang stellt eine fast wortwörtliche Übersetzung des lateinischen Textes dar (vgl. Goehl 2015: 152), im Indikationsbereich wird der Text kürzer gefasst.

\section{Zur übersetzerischen Methode des Juden von Salms}

Einen noch besseren Einblick in die übersetzerische Arbeitsmethode des Juden von Salms bietet uns der Vergleich seiner Übersetzung mit der lateinischen Fassung aus Erlangen (Hs. 674) ${ }^{14}$ und der deutschen Übersetzung in dem ,Leipziger Drogenkompendium' (weiter LDk). Wie schon erwähnt, steht die Edition des LDk nicht zur Verfügung. Außer den in Damms Arbeit publizierten Drogenbeschreibungen hat Johannes Mayer einige wenige (insgesamt 11) Drogenmonographien in einem Artikel (2000) vorgelegt, in dem er sie ihren lateinischen Quellen gegenübergestellt hat. Aus diesen wurde die Monographie über Fenchel ausgewählt. Von der Übersetzung des ,Circa instans'-Textes, die sich im LDk befindet, stellte Damm fest: „Die Bindungen unseres Autors sind noch derart stark, daß manche deutschen Sätze sklavisch wortgetreu aus dem Lateinischen ins Deutsche übersetzt, für uns erst dann ihren Sinn offenbaren, wenn wir sie ins Lateinische zurückübersetzen!“ (Damm 1939: 12)

Wir können nun alle drei Versionen der Monographie über Fenchel/Foeniculum vulgare vergleichen:

Fenicullus calidus est et siccus in secundo gradu. Ex subtili substantia et qualitatibus suis habet virtutem diureticam.

Semen eius, folia et cortices et radices competunt usui medicinae. Cum in receptionibus reperitur receptio maratri, debet poni semen eius. In collyriis debet poni succus radicis vel saltem foliorum eius. Cortices radicis eius non ponuntur sine determinatione. Semen eius in autumno colligitur; per tres annos servatur in multa efficacia. Cortices radicis colliguntur in principio veris; servantur per medium annum.

Contra oppilationem splenis
Feniculus, fenekel, ist heis vnd trocken in dem ij grate, vnde hat rúmende craft von syner behenden substancien vnd qualitatibus, vnd sin same vnd dy bleter vnd rinden vnd worczelen sind bequeme zcu der arcztye. Wanne du findest in den receptis|: nim maratrum, so nim den samen, vnd in den collirijs (nim) das saff der worczelen oder der bleter.

Vnde dy rinden syner worczelen thut man nit in dy arcztye ane entredunge.

Den samen sal sammenen in dem beghinne des herbestes vnd iij mag man yn behalden bi syner macht. Vnde dy rinden von den worczelen sal
Fenicülus ist hytzig vnd dürre in dem andern grade. Sine dogent ist zü dryben. Der same, daz crüt, dye schelen von der würczeln geent in alle arczenie. Vnd wan [man] heyschet feniculum, so meynet man den samen. Plaster zu den aüwen, daz saff von den blettern ist billich, ist dar zü güt, vnde nyt von den würczeln. In anephang septembris leset man den samen. Vnd wert dry jare. Vnd dye schelen von der würczeln leset man in dem anefange prima fera.

Zü swylst des milczes vnd der lebbern, dyssoria vnd drangoria vnd allen bresten des steyns vnd ventositates decoc-

14 Zum Vergleich wird die Edition von Goehl (2015) herangezogen. 
et epatis, stranguariam, dysuriam et vitium lapidis ex calido humore detur aqua decoctionis corticis radicis fenuculi; si ex frigido, detur vinum decoctionis eius.

Feniculaus coctus et comestus valet contra praedicta vel aliquid. Aqua vel vinum decoctionis valet contra dolorem stomachi ex frigiditate vel ventositate; digestionem confortat. Idem valet pulvis eius seminis. Contra leucoflegmatiam fiat decoctio esulae cum succo radicis feniculi, hermodactyli ana scrupulorum duorum; post coletur; et patienti detur in sero, stomacho non saturato vel stomacho ieiuno. Contra pannum oculorum et pruritum succus radicis feniculi in vase aeneo per quindecim dies ad solem ponatur et more collyrii in oculis ponatur. Contra pruritum oculi certum experimentum: conficiatur modicum aloes optimi cum succo feniculi , et ponatur in vase aeneo ad solem per quindecim dies, et ponatur in oculo more collyrii.

(Erlangen, Hs. 674, 74va-74vb, nach Goehl 2015: 91-92) man sammenen in dem beghinne des meyghen vnd eyn halb iar behalden.

Weder dy bestopphunge der milczen vnd der lebern, stranguria vnde dissuria vnde den gebrechen des steynes von heyzer sache: gip das wasser der cochunge der rinden syner worczelen. Jst is aber von kelde, so gip syner cochunge fenikel gesoten vnde geghessen; ist ser gut weder dy voregesprochenen suche. Vnd das selbe wasser oder win alleine czuloset dy wetage des maghen von keldde oder windechtikeit vnde sterket dy douwunge. Das selbe thut das puluer synes samen.

Weder dy leucoflegmanciam: coche in dem saffe der fenikel worczelen esule, hermodactili ana iij drachmas, darnach abelutter is vnde gip is dem lidenden spete; vnde der maghe sal ytel sin, oder vro nuchterne. Weder das tuch uff den oughen vnd iucken: setcze des saffes von fenikel worczelen in eyne erinne oder glesynne vasse in dy sunnen $\mathrm{xx}$ taghe vnde thu is in dy oughen na gewonheit eynes collirij. Weder das iucken der oughen eyn gewis experimentum; conficyre eyn wenigh des besten aloes mit fenikel saffe, vnd setcze is in dy sunnen in eyme erinen vasse $\mathrm{xv}$ taghe vnde darnch thu is in dy oughen na gewonheit.

(Leipzig, Hs. 1224, 124ra-125ra, nach Mayer 2000: 223-225) tio fenicüli ist güt dar zü, oder des samen geszen. Zü dem bresten vorgeschriben waszer decoctio von feniculi ist dar zü güt vnd senfft daz wee des magen vnd ist güt zü boser digestio. Zu flecma mache eyn decoctio myt fenchel würczel saff vnd esula vnd ermodattilis vnd gyb ym daz zü drincken nüchtern. Zü felle[n] in den aüwen vnd gücke vnd fynsternisse vnd zü den auwen, dye dages sehent vnd nachtes nyt, oder die nyt sehent vnd doch den schyn oder glast virnement, nym fenchel würczel saff vnd doe yn eyn coppern geschirre vnd conficere dar vnder aloes cytatrine [!] vnd lege yn dye sonne xv dage vnd dar nach nym des vnd rybe des myt rosen oder fenchel waszers vnd lege ez in die aüwen, vnd ist sicher bewert, daz ez güt ist.

(Zürich, Hs. C 4a: 235ra235rb, Vaňková - Bok in Vorbereitung) 
Wenn wir die beiden Übersetzungen vergleichen, bestätigt schon der Blick auf die ersten Zeilen des Textes aus dem LDk die Worte von Damm über die Abhängigkeit des Textes von der lateinischen Vorlage. Zum Beispiel wird das lateinische Ex subtili substantia et qualitatibus wortwörtlich übernommen, indem die Substantive substantia und qualitatibus in der lateinischen Form verbleiben und nur ex in von und subtili in behend, also gewandt (mhd. behend < bī hende, ,bei der Hand') übersetzt werden. Der Jude von Salms dagegen wählt eine freiere Übersetzung, die dem Sinn entspricht: Sine dogent ist. Ebenso wird das lateinische colirius im LDk beibehalten, auch wenn sich die deutsche Bezeichnung Plaster zu den aüwen, derer sich der Jude von Salms bediente, anbietet. Auch das lateinische certum experimentum wird im LDk getreu übernommen, nur statt certum wird gewis eingesetzt. Der Jude von Salms übersetzt freier und führt die übliche Formel an: vnd ist sicher bewert.

Im Bereich der Indikationen bleiben im LDk einige lateinische Termini unverändert: stranguria (d. h. Strangurie oder Harnzwang), dissuria (Dysurie: ,schmerzhafter Harnabgang, erschwerte, gestörte und schmerzhafte Blasenentleerung ${ }^{6}$ ), leucoflegmacia (WeiBe Wassersucht). Auch der Jude von Salms verwendet die lateinischen Bezeichnungen beider Krankheiten (dyssoria, drangoria). Leucoflegmatia vereinfacht er jedoch zu flegma. Unter Flegma versteht man aber eher den Schleim, einen der vier Säfte, die - entsprechend der Humoralpathologie - der menschliche Körper in sich enthält, sodass diese Übersetzung verwirrend wirkt.

Im LDk kann man das Bemühen beobachten, die lateinischen Termini ins Deutsche zu übertragen: Statt ventositates (auf Deutsch ,Blähungen“, d. h. übermäßige Ansammlung von Gas in Magen und Darm) schlägt der Übersetzer die Bezeichnung windechtigkeit vor, die auf das lateinische ventus (Wind) zurückgeht. Der Jude von Salms bevorzugt in einigen Fällen eher die lateinischen Fachwörter: Nicht nur ventositates, sondern auch boser digestio (d. h. schlechte Verdauung) stellt er als Indikationsbereich vor. Im LDk findet man in diesem Fall die verbale Ausdrucksweise: sterket dy douwunge. Der Jude von Salms führt auch konsequent das lateinische decoctio an, im LDk steht aber das deutsche cochunge, das auf das ahd. cohunga zurückgeht. Heute wird das Wort „Kochung“ nicht gebraucht: Stattdessen wird eine präfigierte Form, also Abkochung, bzw. Absud oder Dekokt verwendet.

Das Streben nach einer genauen Wiedergabe des Originals in dem ,Leipziger Drogenkompendium‘ wirkt zwar auf der einen Seite ein bisschen hölzern, auf der anderen Seite ermöglicht es, alle Bedeutungen des Originals zu erfassen. So wird nach der lateinischen Vorlage in der Aufforderung nim maratrum (lat. receptio maratri) die ursprünglich griechische Bezeichnung des Fenchels márathron verwendet, die ins Lateinische als maratrum übernommen wurde. Der lateinische Text weist darauf hin, dass mit maratrum nur der Samen des Fenchels gemeint wird: Cum in receptionibus repetitur receptio maratri, debet poni semen eius. Die Übersetzung im LDk Wanne du findest in den receptis $\mid$ : nim maratrum, so nim den samen entspricht also dem Hinweis im lateinischen Original. Dagegen hat der Jude von Salms den Terminus maratrum gemieden und durch fenikulum ersetzt. Dadurch ist aber der Hinweis, dass Fenchelsamen als marathrum bezeichnet wird, verloren gegangen.

Interessant ist auch, dass der Jude von Salms die Gewichtsangaben, die sich im lateinischen Text befinden und die auch im LDk vorliegen, oft - nicht nur in der Monographie über Fenchel - ausgelassen hat, vgl.: 
Contra leucoflegmatiam fiat decoctio esulae cum succo radicis feniculi, hermodactyli ana scrupulorum duorum... (E)

Weder dy leucoflegmanciam: coche in dem saffe der fenikel worczelen esule, hermodactili ana iij drachmas... (L)

$\mathrm{Zu}$ flecma mache eyn decoctio myt fenchel würczel saff vnd esula vnd ermodattilis (Z).

Während im lateinischen Text scrupulus als Gewichtsangabe angeführt wird (ein Scrupulus entsprach etwa 1,24-1,25 Gramm), wurde im LDk das Gewicht in Drachmen (aus dem Griechischen übernommen, bezeichnet eine Handvoll) angegeben. Da eine Drachma etwa 3,7 Gramm entspricht, lässt sich ableiten, dass die Gewichtsangaben in der lateinischen Version und der Übersetzung im LDk nicht übereinstimmen (was jedoch beim Abschreiben der Texte ein häufiger Fall war).

\section{Zusammenfassung}

Die angeführten Beispiele aus dem Kompendium des Juden von Salms zeigen, dass es sich auf keinen Fall um eine wortwörtliche, von der Vorlage abhängige Übersetzung des ,Circa instans' handelt, wie man es in dem ,Leipziger Drogenkompendium‘ beobachten kann. Der Autor geht mit der (unbekannten) lateinischen Vorlage viel freier um, er kürzt oft auch den Text, wenn er der Meinung ist, dass er das Wesentliche erfasst hat. Besonders im Bereich der Indikationen fasst er sich manchmal zu kurz, sodass eher eine Liste der Anwendungsbereiche der Droge vorliegt, ohne eine genauere Beschreibung der Herstellungsverfahren der entsprechenden Medikamente. Diese Vorgehensweise ist wahrscheinlich nicht nur auf den Zeitdruck, unter dem der Jude von Salms angesichts seines hohen Alters gearbeitet hat, zurückzuführen, sondern auch auf den Zweck seiner Übersetzung. Diese sollte in erster Linie als Informationsquelle dem Grafen Johann von Sponheim dienen und nicht als eine Anweisung für die Praxis. Nichtsdestoweniger ist das Bemühen deutlich, dem Leser alle wesentlichen Informationen zu vermitteln, so dass das ,Circa instans“ im ,Medizinischen Kompendium“ des Juden von Salms zweifelsohne eine einzigartige Überarbeitung dieses Arzneidrogenbuches in der deutschen Sprache darstellt.

\section{Quellen und Literatur}

\section{Quellen}

Hs. C4a, Zürich: Universitätsbibliothek.

Hs. 674, Erlangen: Universitätsbibliothek.

Hs. 1224, Leipzig: Universitätsbibliothek. 


\section{Sekundärliteratur}

Bok, Václav (2014): Einige Bemerkungen zum sog. Juden von Solms und den Handschriften seines Werkes. In: Vaňková, Lenka (hrsg.): Fachtexte des Spätmittelalters und der Frühen Neuzeit. Tradition und Perspektiven der Fachprosa- und Fachsprachenforschung. (Lingua Historica Germanica, 7). Berlin: Walter de Gruyter, S. 65-73.

Damm, Walter (1939): Die einzige bisher bekannte deutsche Fassung des Buches Circa instans (de simplicibus) nach einer Handschrift des 15. Jahrhunderts. (Leipzig, Universitätsbibliothek Nr. 1224). [Hamburg: Preilipper].

Goehl, Konrad (2015): Das Circa Instans. Die erste große Drogenkunde des Abendlandes. (DWV-Schriften zur Medizingeschichte, 17.) Baden-Baden: Deutscher Wissenschafts-Verlag.

Holler, Fritz-Heinz (1940): Das Arzneidrogenbuch in der Salernitanischen Handschrift der Breslauer Stadtbibliothek (Nr. 1302) (Diss. math.-nat., Berlin). Würzburg: K. Triltsch.

Keil, Gundolf (1961): Die ,Cirurgia‘ Peters von Ulm. Untersuchungen zu einem Denkmal altdeutscher Fachprosa mit kritischer Ausgabe des Textes. Ulm: Süddeutsche Verlagsgesellschaft.

Keil, Gundolf (1978): ,Circa instans'. In: Ruh, Kurt [et al.]: Die deutsche Literatur des Mittelalters. Verfasserlexikon, Bd. 1 (2. Aufl.). Berlin - New York: Walter de Gruyter, Sp. 1282-1285.

Keil, Gundolf - Dilg, Peter (1991): Kräuterbücher. In: Lexikon des Mittelalters, Bd. V, München Zürich: Artemis-\&-Winkler-Verlag, Sp. 1476-1480.

Mayer, Johannes Gottfried (2000): Das „Leipziger Drogenkompendium“ (Leipzig, Universitätsbibliothek, Cod. 1224) und seine Quellen ,Circa instans', ,Aggregator“ (Pseudo Serapion), ,Macer floridus‘ (bzw. , Älterer deutscher Macer'), ,Liber graduum‘ (Constantin) und ,Liber iste‘. In: Goehl, Konrad - Mayer, Johannes Gottfried (hrsg.): Editionen und Studien zur lateinischen und deutschen Fachprosa des Mittealters. Festgabe für Gundolf Keil zum 65. Geburtstag. Würzburg: Verlag Königshausen \& Neumann, S. 207-263.

Mayer, Johannes Gottfried (2004): ,Leipziger Drogenkompendium‘. In: Wachinger, Burghart [et al.]: Die deutsche Literatur des Mittelalters. Verfasserlexikon 11 (2. Aufl.). Berlin - New York: Walter de Gruyter, Sp. 912-915.

Mayer, Johannes Gottfried (2014): Das ,Leipziger Drogenkompendium‘ und der ,Gart der Gesundheit‘. Ein Vergleich. In: Vaňková, Lenka (hrsg.): Fachtexte des Spätmittelalters und der Frühen Neuzeit. Tradition und Perspektiven der Fachprosa- und Fachsprachenforschung. (Lingua Historica Germanica, 7). Berlin: Walter de Gruyter, S. 133-142.

Palmer, Nigel F. - Speckenbach, Klaus (1990): Träume und Kräuter. Studien zur Petroneller ,Circa instans'-Handschrift und zu den deutschen Traumbüchern des Mittelalters. Köln - Wien: Böhlau Verlag.

Schnell, Bernhard (in Zusammenarbeit mit William Crossgrove) (2003): Der deutsche „Macer“ (Vulgatfassung) - mit einem Abdruck des lateinischen Macer Floridus „De viribus herbarum“. Tübingen: Max Niemeyer Verlag.

Schnell, Bernhard (2008): Varianz oder Stabilität. Zu den Abschriften deutscher mittelalterlicher Medizinliteratur. In: Zeitschrift für deutsche Philologie (2008), Sonderheft zum Bd. 127: Der Schreiber als Dolmetsch. Sprachliche Umsetzungstechniken beim binnensprachlichen Texttransfer in Mittelalter und Früher Neuzeit, S. 27-47.

Vaňková, Lenka (2017): Krankheitsbezeichnungen in der Chirurgia des Juden von Salms. In: Jörg Riecke (hrsg.): Sprachgeschichte und Medizingeschichte. Texte - Termini - Interpretationen. (Lingua Historica Germanica, 16). Berlin - Boston: Walter de Gruyter, S. 47-62.

Ventura, Iolanda (2009): Ps. Bartholomaeus Mini de Senis, Tractatus de herbis (Ms London, 
Zur deutschen Übersetzung des ,Circa instans' im ,Medizinischen Kompendium des Juden ...

British Library, Egerton 747). (Edizione Nazionale “La Scuola Medica Salernitana”, 5). Firenze: SISMEL.

Ventura, Iolanda (2016): Medieval Pharmacy and Arabic Heritage: The Salernitan Collection Circa instans. In: Micrologus XXIV: The Impact of Arabic Sources on Divination and the Practical Sciences in Europe and Asia, ed. Agostino Paravicini Bagliani. Firenze: SISMEL, S. 339-401.

Wölfel, Hans (1939): Das Arzneidrogenbuch Circa instans in einer Fassung des XIII. Jahrhunderts aus der Universitätsbibliothek Erlangen (Diss.). Berlin: Friedrich-Wilhelms-Universität.

Prof. PhDr. Lenka Vaňková, Dr. / lenka.vankova@osu.cz

Ostravská univerzita, Filozofická fakulta, Katedra germanistiky,

Reální 5, 70103 Ostrava 1

Prof. PhDr. Václav Bok, CSc. / bokovi@web.de

Ostravská univerzita, Filozofická fakulta, katedra germanistiky,

Reální 5, 70103 Ostrava 1 